\title{
ARTIFICIAL INTELLIGENCE: WAY FORWARD FOR INDIA
}

Sunil Kumar Srivastava (Dhttps://orcid.org/0000-0002-7752-1328

Government of India, Ministry of Electronics and Information Technology, New Delhi, India

\begin{abstract}
Artificial Intelligence (AI) is likely to transform the way we live and work. Due to its high potential, its adoption is being treated as the fourth industrial revolution. As with any major advancement in technology, it brings with it a spectrum of opportunities as well as challenges. On one hand, several applications have been developed or under development with potential to improve the quality of life significantly. As per a study, it is expected to double the annual economic growth rate of 12 developed countries by 2035. On the other hand, there is a possibility of loss of jobs. As per the available reports, the loss of jobs during the next 10-20 years is estimated to be $47 \%$ in the US, $35 \%$ in the UK, $49 \%$ in Japan, $40 \%$ in Australia, and $54 \%$ in the EU. In the era of globalization, no country can isolate itself from the impact of the advances in technology. However, the benefits can be maximized and losses can be minimized by putting necessary infrastructure and policy in place. Though several countries have decided their strategy for AI, India has not yet formulated its strategy. This article reviews the international as well as national scenario and suggests way forward for India.
\end{abstract}

Keywords: Artificial intelligence, India, way forward, applications, policy

Manuscript first received: 2017/09/22. Manuscript accepted: 2018/03/15

Address for correspondence:

Sunil Kumar Srivastava. Government of India, Ministry of Electronics and Information Technology, New Delhi, India.

E-mail: sks@meity.gov.in 


\section{INTRODUCTION}

Artificial Intelligence (AI) is concerned with understanding the nature of human intelligence and designing intelligent artefacts which can perform the tasks which, when performed by humans, are said to require intelligence. Any major advancement in technology brings with it a range of opportunities and challenges. While AI is likely to bring substantial economic growth, it is being predicted that a number of jobs would be lost due to the automation. Therefore, it is necessary to put required policy and infrastructure in place.

Though the field of AI has been an area of extensive research since the term was coined in 1956, it has recently only led to large-scale deployment of intelligent applications for different domains and tasks. The works in the late fifties and early sixties were in the direction of development of general techniques, which could be applied in several domains. The results were not very encouraging and it led to the first winter of the field, which started in the late sixties and continued till the late seventies.

It was realized that the knowledge of the domain plays an important role and therefore, the focus of the research was on representing and using knowledge. The systems developed were called knowledge-based systems. MYCIN, a KBS using rules for representing knowledge of the experts was used successfully in the medical domain. A number of such systems were developed in other domains. As these were based on the knowledge of human experts, these were called expert systems. Several companies captured the opportunity.

However, by the late eighties, it was found that the rules were often brittle and did not work in practical applications which required incorporating a large number of rules to deal with various scenarios. When several companies failed in their efforts in applying the technology, there was general disappointment in the industry. The second winter of AI started in the late eighties and continued till the mid of the first decade of the current century when high computational power machines and storage became easily available and affordable. It was fuelled by the development of certain algorithms such as deep learning. Large-scale deployment could be easily possible on mobile phones using Internet. It may be noted that the periods of the two winters mentioned above are not precise due to obvious reasons.

The present sharp rise in the number of AI applications is due to several factors. Firstly, during the last few decades, the computational power and storage capacity of computers has been increasing while the cost has been decreasing due to the advances in the electronics used in complex digital circuits. This has made computing more and more affordable. One of the main requirements for application of AI is the availability of high computing power, large memory and storage, which have now become affordable. The cloud technology has supported it further. It has made it possible to deploy AI applications without high upfront cost.

The second important factor is the advances in the AI techniques, such as deep neural networks, etc. Though the concepts such as machine learning, neural networks, etc, have been there since the sixties, the sophistication of algorithms has increased substantially after several decades of research. The progress in machine learning has made significant impact in the practical applications of AI. This has also led to the debate on the Master Algorithm - an algorithm which can learn any knowledge from the given set of data (Domingos, 2015). Another area of AI where significant progress has been made, is natural languages processing used in machine translation, user-interface, etc. Using these advances in the technology, a number of applications were developed to assist people in natural language. These were called chatbots and were used for the tasks such as answering the questions of 
customers. Some companies such as Google, Microsoft developed chatbots to perform several other tasks on behalf of the users. These are known as virtual assistants.

The third factor is the generation, storage, transmission and processing of high volume, high speed, and high variety data due to the technological advances including Internet of Things (IOT), low cost sensors, high speed communication networks, and mobile devices. High volume of data can provide several types of insights, which have not been possible so far. For example, Google is using large corpora of texts in several languages for use in machine translation between two languages. It breaks the text into phrases and uses it to learn the translations of the phrases between two languages.

Industry has recognized the value of AI and the companies have re-aligned their strategy to it. Google has adopted first AI policy. It is exploring the application in every area of its business. In 2014, it announced that it would become an AI firm by 2024. It may be noted IT companies are achieving higher and higher ranks in brand values over the last few years. In 2016, Apple became the most-valued brand but replaced by Google in 2017.

There has been an ongoing debate on the risks associated with AI. Several personalities like Stephen Hawking, Elon Musk, etc have raised this issue from time to time. The most cited risk associated with AI is the existential threat. It has been argued that AI development may lead to creation of systems which can destroy humanity itself. Such a situation may arise when a system is created which can't be controlled by humans. At the same time, several thinkers have expressed their concern that this expected risk may stop the development of AI applications and thus depriving the people from the benefits of AI. In order to highlight the need of research on the safety issues, a large number of scientists have come together and signed an open letter which calls for a balance by advocating development of new applications of AI while avoiding pitfalls.

An analysis of the major economies (Purdy \& Daugherty, 2016) shows that the GDP growth has been stagnant or has declined in several developed countries during the past few decades. The average GDP growth of Japan, US, UK, France, Italy, Germany was 3.0 in the eighties, 2.1 in the nineties and 1.1 during the first decade of the current century. For quite some time, capital and labour have been seen as the factors of production. However, studies show that the capital efficiency rate has been declining. Further, the working age population is not increasing in many developed countries. The net effect is the marginal GDP growth in the developed countries. Accenture has done an analysis of 12 major economies and have predicted that the adoption of AI has the potential to double the economic growth rate by 2035 .

In view of the recent advances in the area of AI and its potential impact on the job opportunity, it has become necessary for every country to prepare an action plan to take the benefits of the opportunity and deal with the challenges. It needs to identify the priority areas for investment in technology development to build AI-based solutions. At the same time, it should also take steps to prepare the workers to take up the new types of jobs which may emerge. It is not possible for any country to keep itself untouched from the impact but it can deal with it well by putting necessary infrastructure and policy in place (U.S. Government, 2016).

Several countries have already formulated their policy for AI (U.S. Government, 2016; House of Commons Science and Technology Committee, 2016; State Council of China, 2017; Government of South Korea, 2017; Benner, 2017). India can't afford to be left behind. It missed the benefits of the first and second industrial revolutions due to political reasons. However, it could gain the benefits of the revolution due to information technology using the talent available in the country. It has not only made wealth by the export of software but could provide jobs to millions of educated people. This advantage is 
at risk today since most of the companies are automating the processes using AI technology. Therefore, the country has to make it ready to gain from the opportunity and face the challenges due to AI.

The problem is reflected in the recent news from the business world. Recently, Infosys announced that it has released 8000-9000 employees during the last one year due to automation of jobs. Out of that few hundreds were being retrained for new types of jobs. These employees who have been released and are not being re-skilled are likely to lose their jobs. Similar trends can be seen in other companies.

Though the number of laid off employees may be exaggerated, it is time that all the stakeholders take a note of this. Even if the number of employees laid off is small, we need to explore the ways to retrain them and support them in the phase of transition of job so that they can deal with the situation in an effective way.

In view of the above, it is necessary for India to formulate a well-considered policy. The report presents an overview of the area and suggests way forward for India. It looks into the way the technology is being used in various countries across the world and examines the status of AI applications in India. It also suggests what need to be done with respect to Applications \& Infrastructure; Regulations \& Policy; Research \& Development; and Human Resource Development.

Though the private sector has been doing quite well in the development of AI applications, public sector needs to be sensitized and supported to make use of the technology. The applications will require investment in computing infrastructure as well. Regulations need to be reviewed in the sectors like transport where AI applications are likely to be used in near future. Policy is needed for making government-owned data available to the developers. As in other countries, government has to support R\&D in basic research and in the areas requiring long-term research. Human resource development is needed to create required manpower to work on AI applications development and deployment. Also, training infrastructure needs to be created for the workers displaced by AIdriven automation.

\section{AI APPLICATIONS - INTERNATIONAL SCENARIO}

In order to assess the current level of adoption of AI technology, there is a need to look into the applications which have been developed across the world. Applications have been developed in almost every walk of life (Nassiri-Mofakham, 2017). The following paragraphs discuss the applications which have been deployed or are at the advanced level of development. The examples given below are for illustration purpose only. As new applications are being developed every day, there may be many more types of applications which might not be listed here.

\section{Healthcare}

AI has been used in the domain of healthcare for quite some time. According to a report, there are more than 100 startup companies working on the application of AI in healthcare domain (From Virtual Nurses To Drug Discovery, 2018). Some applications have already been deployed whereas a large number of these are under development in several countries. Using the advances in natural language processing techniques, several conversational applications have been developed. Virtual nurse is an application which is being used in several types of environments. It can perform the tasks which are normally performed by a human nurse. Molly, a virtual nurse developed by 
the startup, Sense.ly assists the patients in managing the chronic conditions after the treatment or between the follow-up consultations. ${ }^{1}$

The ability of AI algorithms to locate the relevant information has been found to be of great use. Watson for Oncologists developed by IBM helps a doctor in finding the relevant material from a large number of papers/documents which could be of use in the case at hand. It analyzes both structured and unstructured data. If the present case is similar to any case reported in any publication/document and a particular treatment plan has been found to be effective, the information reported may be useful for the treatment in the present case (IBM, 2018).

AI is helping in providing personalized treatment to the patients. Every patient is a different individual and may need a different treatment. Further, a disease may have thousands of subtypes requiring different treatments consisting of a combination of drugs. For instance, it is being realized that cancer has thousands of subtypes and each subtype requires different combination of drugs for effective treatment. On the other hand, pharmaceutical companies rely on large-scale randomized clinical trials for testing new drugs. This limits the number of cases, it would be effective. This is why treatment often requires a trial and error approach. Once we have sufficiently large database of cancer cases, it becomes possible to find cases similar to the case in hand and there is a good probability that the treatment found to be effective in the earlier cases would be effective in the present case too (Tenenbaum \& Shrager, 2011).

Some companies are developing AI-based systems which can provide consultation. It is especially useful in non-critical conditions or in the situation when human doctor is not available at all. An example is Babylon developed by Babylon Health (Babylon, 2018). It provides consultation to the user based on the symptoms reported. It asks the user few simple questions in spoken natural language and the user can answer in natural language. It searches a large database of symptoms and provides the appropriate medical advice. In case it finds necessary, it advises the patient to approach the doctor immediately.

\section{Education}

Intelligent tutoring systems have been developed since the eighties. Several applications have been in use for quite some time. With the advances in AI techniques such as natural language processing, etc, it has become possible to develop a number of new applications. As education itself is crucial for growth in several domains, the net impact of AI in education would be quite high. The applications of AI in education include answering the queries of the students, asking questions and providing feedback, assessment of narrative answers. A study has predicted that AI would transform education during the next 4-5 years (Kanejiya, 2017).

Apart from AI, several new technological advancements are changing the scenario of education. With smart phone, the material has become more accessible to a wider class of people. Students can participate in the discussions. Learning outcomes are also improving with the use of learning management systems. Massively online open courseware (MOOC) has become popular with time.

It has become possible to create bots which appear like a human. In an experiment, a professor at Georgia Tech created an AI-powered system to work as his teaching assistant to assist in a course which was being taught by him. It interacted with the students just like a human teaching assistant (McFarland, 2018). It assessed the assignments of the students for grading and answered their queries in natural language using email. The students could never realize during the entire semester that they were interacting with a system and not a human until they were told so by the professor himself.

1 Sensely Molly: Virtual Nurse. http://Sense.ly [Accessed February 20, 2018] 
With AI, it has become possible to assess the subjective answers given by the students. Use of machines in assessing subjective answers results in substantial saving of time and brings uniformity in the assessment. Bots are being used to assess the subjective answer given by the students appearing in GRE test administered by ETS (ETS, 2018). In one part of the test to assess the reasoning ability of the student, the students are supposed to give narrative answers. Till now, this was being assessed by two human experts to reduce the possibility of any error. After testing the performance of the application over a long duration of time, ETS has now replaced one of the two human experts by a bot.

Despite the availability of a large amount of digital learning material, the use of computerbased education has remained limited due to lack of the possibility of personalization. A human teacher personalizes the content depending on the needs of the student. Every student has a different level of knowledge and aptitude. Therefore, the same content for every student does not work. If the student has not been able to understand the current topic, the system should not proceed to the next topic. Similarly, if a student already has knowledge of the topic, the content becomes repetitive and the student gets disinterested. Personalization has become possible using AI. Such a system can adapt the content depending on the requirements of the student. It assesses the performance of the student and tailors the content accordingly.

\section{Cyber Security}

The rapid increase in the size and complexity of the virtual world has led to a continuing war between cyber attackers and security service providers. Each side is trying to develop more sophisticated techniques and tools. Cyber space is a dynamic environment where situation keeps on changing rapidly and can't be predicated with certainty. As AI systems have the flexibility to respond to the changing environment, its use in increasing in all the stages in the cyber defence chain viz. early warning, prevention, detection and, response (Wirkuttis \& Hadas, 2017).

AI reduces the human intervention by the automation of the processes. The conventional cyber security systems are slow due to the need of human interaction in several steps. As any delay can cause significant damage, it has to be kept at the minimum. The loss is not limited to financial damage only. In case of denial of service attack on a hospital, the access to the health records of the patients is slowed down. This may become fatal for a patient requiring immediate intervention.

With AI technology, it has become possible to create artificial police agents for monitoring the entire network. It uses intelligent agents to do this work. An intelligent agent is an application which has the ability to sense, reason and act autonomously. These agents can interact with each other to collaborate to perform a task. Sufficient number of intelligent agents can be deployed to monitor the network to detect the malicious activities in a decentralized way.

However, despite all these developments, it is not yet possible to replace human by machine in the defence against cyber attacks. Both human and machine have their strengths and weaknesses. If both combine their strengths, the success rate is higher than in case of individual cases.

\section{Law}

Several AI-based applications have been developed and services are being offered in the domain of law. AI-based systems have been developed for legal research which involves finding the similar precedent cases for deciding the present case or making arguments in the present case. The companies like Lexus and Westlaw have been offering applications based on keyword based matching for quite 
some time. However, it was realised that the results have high number of positive false as well as negative true. This happens as keyword based searching is based on literal meaning of the words and not on its interpretation. AI based technique gives better result as it searches using the content of the case (Houlihan, 2017; Mills, 2016; How artificial intelligence is transforming the legal profession, 2018; Cessna, 2015; RAVN Systems, 2016).

Conversational applications have been developed to advice in legal matters in an interactive way. For instance, a chatbot namely, DoNotPay helps people in filing appeals against issuing of parking tickets if there are valid reasons. It has been used in London and New York in 375,000 cases within a short period for filing appeals involving 7.2 million pounds in fines. It asks the user simple questions and advises on the issue. After interaction, it also generates an appeal using the information given by the user in response to various questions. The service is being provided free of cost. Similarly, an application was developed to provide legal advice to the people seeking asylum in the US, Canada and the UK. After success of these applications, 1000+ bots have been developed for a range of topics for the people in 50 States in the US and the UK (DoNotPay, 2018).

AI systems have been developed for contract analysis, especially in corporate sector. Contract analysis involves going through a large number of contract and related documents used over a period of time to find the significant clauses. Often the time required for this is too long and it may not be practically possible to complete the task in many situations. In such a situation, it is possible to use AI-based systems to go through the documents and highlight the most relevant clauses. Lawyers can focus on these clauses rather than going through all the documents which may not be possible within the limited time and resources (e-Brevia, 2018; Kira System, 2018).

\section{Finance}

Chatbot is being used by banks for performing simple tasks such as activation of accounts or balance checking, etc. It helps the customers who are not fully familiar with IT systems and would like to interact in natural language. Chatbot asks the customers questions in natural language and performs the needed tasks. Some investment consulting firms are also using chatbot to interact with the customers. The chatbot asks the customer some questions, which vary from customer to customer, to get the basic information on the needs of the investor and then generates plans based on the market trends, etc. The plans can be reviewed from time to time as and when further information is available. Though this can be done by a human adviser, chatbot performs it quickly and accurately [Yacoub, 2018). State Bank of India, HDFC Bank, ICICI Bank and Axis Bank have started using AI-based applications for providing customer services in India.

Most of the banks have policy of upgrading the IT solutions including AI-based applications for customer service and use of robots in the processes. Earlier applications were limited to providing the customers information in natural language. Now these applications complete some of the banking transactions on behalf of the customers. Some of the governments have adopted policy to promote applications of AI in the banks. For instance, Government of Singapore has announced a grant of \$19.9 millions for the banks located in Singapore to promote AI and data analytics.

As vast amount of data on stock trading is available publically, it is an excellent area for the use of AI. Sentinent Technologies Inc. is developing an AI system for taking decisions in stock trading (Satariano, 2018). It has a team of engineers who have worked for Amazon, Apple, Google, Microsoft, etc. The other firms which are exploring the idea of using AI in hedge fund include Wealthfront and RBS, etc (Desai, 2018). 
Some applications have been built to help in the preparation of tax returns. An example chatbot is AskMyUncleSam. As the US tax code is complex with a series of deductions, etc, a tax payer often goes to tax return preparer. The chatbot guides the tax payer in this process. It goes through the data on payments received, investment loss \& gains from brokerage accounts, etc and finds all the possible deductions and liabilities. It answers the queries of the user using its knowledge base of tax code, litigation cases, etc (Ogunsanya, 1998).

\section{Information Browsing}

Often, AI is embedded in the normal systems of daily use and is invisible to the users. For example, Google search engine uses sophisticated AI algorithms to predict the content that the user may be interested. It brings the most relevant documents at the top which makes it the best search engine (Brin, 1998). As the volume of the content is increasing on Internet, such search engines are of great help to the users.

Apart from information retrieval, search engine companies use the information on the user preferences to decide the advertisements to display. Google monitors the news items being read by the user and uses this information to decide the news of interest to the user to display.

Google also analyzes the data collected from the browsing history of a person to decide the advertisements of interest and the same is displayed while the user is browsing. It has been found quite effective. Google generates revenue from the number of hits made by the users (Google Inc, 2018).

\section{Transport}

Self-driving car is a high-potential application of AI. Several companies including Google, Uber, and Tesla are testing their models on the roads. In Singapore, driver-less bus is being run under trial. So far very few accidents have been reported and the analysis shows that probability of accident with driverless cars is less than human driven cars. It is expected that the number of accidents by selfdriving cars will be much smaller than the human-driven cars (Jiang, 2015).

Self-driving cars use light detection and ranging (LiDar) technique which uses laser beams to create 3D image of the physical world around the car. It uses laser beams to calculate the distance, speed and shape of the moving objects like another car, pedestrians, etc. Apart from roads, the technology can be used by those who can't walk due to physical limitations. Several companies are competing with each other in this area of technology.

There has been a debate on whether these cars should be allowed on the road in view of the danger to the safety of the people. Regulations have to address several issues. For example, who will be responsible if an accident happens - the owner of the car or the company which has made it? Some States in the US are formulating regulations to deal with these issues. The companies are going ahead and have definite plans to make it available to the people in near future.

\section{Virtual Assistants}

A virtual assistant helps user in performing tasks or perform the tasks on their own in an autonomous way. Several virtual assistants have been developed. The examples are Google's 
Assistant ${ }^{2}$, Siri ${ }^{3}$, Cortana $^{4}$, and Alexa ${ }^{5}$ from Google, Apple, Microsoft, and Amazon, respectively. User can use natural language to interact with a virtual assistant. As one can also interact with spoken words, an illiterate or blind person can also use it.

Virtual assistants perform a variety of tasks including streaming music, playing audio clips, controlling home devices, managing schedules, purchasing using e-commerce, etc. These assistants are becoming more and more useful due to the advances in natural language processing and speech recognition. This makes it useful to a common person without any specific training.

Speech recognition makes it possible to use even while driving, etc. The vendors are trying to put these assistants in vehicles. Siri has already been integrated with some cars. Nissan is integrating Microsoft's platform into its cars. Similarly, Hyundai is integrating with Alexa and Google Assistant.

\section{E-Commerce}

There are a number of product and service recommender systems such as the one used by Amazon on its shopping portal. It keeps a track of which items have been purchased by the people over a period of time and identifies certain patterns which are used to decide the products and services of interest to the user. These patterns are not fixed and hard coded in the system but are created using machine learning techniques (Alluhaidan, 2018).

Chatbot is being used to order products and services online. A chatbot has been developed to order coffee from Starbucks. The customer can order coffee using spoken natural language describing the type of coffee, etc and the order is sent to the nearest Starbucks unit. The payment is made automatically using the pre-registered credit/debit card (Sarah Perez, 2018).

\section{Customer Care}

AI systems have been developed for customer care in several sectors. The systems use natural language to interact with the customers. Though chatbot has been used for customer care for a long time, it has become more useful with better natural language processing and speech recognition. If the customer remains unsatisfied, it is handed over to a human executive. In order to reduce the cost, companies have used IVRS to handle simple cases but the customers find that they have to go through a number of questions to get the information. On the other hand, a chatbot takes user's input in a natural language and asks only the relevant questions for addressing the issue. During the dialogue, chatbot takes context into account. If the discussion relates to a particular product or service, it uses and recognizes the pronouns used for the same.

Chatbot takes into account the emotion of the user as well. Amelia, a customer service agent from IPSoft uses sentiment analysis to understand the mood of the customer to adapt its response accordingly, for instance, when it faces an angry customer, it uses words / phrases for reassurance. As all the conversations are stored, it picks up the related conversation to begin with. This, obviously, has advantage over human executives who can't retrieve the related conversation from the database quickly. If the issue remains unresolved by the chatbot, the call is transferred to a human executive (Amelia, 2018).

2 https://assistant.google.com/ [Accessed February 20, 2018]

3 http://www.apple.com/in/ios/siri/ [Accessed February 20, 2018]

4 https://www.microsoft.com/en-in/windows/cortana [Accessed February 20, 2018]

5 http://www.alexa.com/ [Accessed February 20, 2018] 
Amazon has recently implemented an automated sales system in its store in Seattle. It monitors each customer and captures the information on the items being picked up by him for purchase. Once the customer has collected all the relevant items, he or she can walk out and the payment is deducted from the pre-registered credit/debit card. Such a system is likely to reduce the human sales force from the stores (Amazon Go..., 2018).

\section{Energy}

AI is being used in energy sector in several ways (Jucikas, 2018). Making energy clean, affordable and reliable has been recognized essential for fighting against several problems including poverty. Google has applied AI successfully in reducing the energy usage by $40 \%$ which means several millions of dollars. Google used the technology used in DeepMind for predictions on loads at different points and controlling equipments efficiently, accordingly.

In view of this success, National Grid in the UK is working with DeepMind team to explore the opportunities. DeepMind aims to cut the national energy bill by $10 \%$ by balancing energy supplies to the national grid. The extra power generated by the operators but not used leads to the wastage of power. This is becoming possible due to the availability of past data which is used for learning and predictions.

IBM is working with the Department of Energy in US for solar energy prediction. IBM has developed the technology by several forecasting models and integrating data on weather, environment and atmospheric conditions, etc collected from different sources such as weather stations, satellites, sensor networks, etc. IBM claims that the model developed is $30 \%$ more accurate than the best available solar forecasting model. After its successful application in solar energy, IBM is exploring the possibility of using it in wind and hydro-power plants.

AI has also been used to understand the consumer behaviour for prediction of load pattern. In order to gather this data, the energy provider companies are installing smart meters which send information on the usage periodically. As different persons and different communities have different consumption patterns, it is important to gather this data for prediction of loads.

\section{Business Strategy}

The prediction capability of AI-based solutions can be used in deciding business strategy (Agrawal; Gans \& Goldfarb, 2018). A company uses predictions about the customers and market conditions to decide where to focus. Once more and more accurate predictions are available, the company may decide to use different strategy. For instance, the predictions about the customers can be used by a company to bring different types of products and services. One example of use of AI for predictions is that by Amazon which recommends certain products when someone shops on the platform. More extensive data on the customers can help in making the decisions on where to store the products and in what quantity.

\section{IMPACT ON JOB OPPORTUNITY}

The benefits of AI come with a price. According to the studies on the impact of AI, the job loss during the next $10-20$ years is estimated to be $47 \%$ in the US, $35 \%$ in the UK, $49 \%$ in Japan, $40 \%$ in Australia, and 54\% in the EU (Government of Canada, ). Though the sources may differ in the figures for the jobs at risk, everyone agrees that a number of jobs are at risk of being lost across the world in the next few decades. In this sense, it is a disruptive technology (McKinsey Global Institute, 2015). At the same time, experts are of the view that a new range of jobs would emerge during the process. 
Though wide-spread use of AI may lead to the loss of a large number of existing types of jobs, industry leaders feel that ultimately it would lead to net increase in the number of jobs available. The emerging jobs will be of different types just like different types of jobs emerged during the first industrial revolution. At that point of time, a large number of manual jobs were lost due to the use of machines but a whole new range of jobs was created and millions of people were employed to work with machines. The loss of jobs led to riots in the countries but ultimately, it was realized that the use of machines was beneficial for the society (Frey \& Osborne, 2015).

The newly created jobs may require different sets of skills. Sometimes, the needed skills may not be available and therefore, the workers would have to be re-trained. The existing jobs may also change in terms of the requirements of skills (U.S, 2016). This means many workers would go through a transition phase. They need to be supported during this phase. Also, a good number of professionals would be needed to develop and deploy AI applications.

However, several experts are of the view that the job opportunity in India may not be affected so much due to AI. India is a growing fast economically in comparison to other countries. The growth will provide new avenues of employments. Further, with its talented engineers, India can get a major share of AI-based applications development market.

In this scenario, it is important to identify the new opportunities where AI can be used and prepare the workforce to meet the challenges due to the change in the nature of jobs. On one side, we need to develop good quality AI-powered applications quickly enough so that we don't lose the market within and outside the country. If the applications are delayed or lack in the quality, there may not be takers. On the other side, we need to re-train the workers to take up the new jobs. Also, we need to deal with other issues such as legal, ethical, etc. which are posed by the development of new products and services. That is how Europeans created wealth during the first two industrial revolutions and became more prosperous in comparison to the countries which could not make use of the opportunities as in the case of India.

\section{GOVERNMENT INITIATIVES}

Several countries have formulated their strategy to gain from the potential of the artificial intelligence technology while addressing the challenges, especially the loss of jobs. The Government in these countries have formed Committee of Experts to assess the potential and challenges of the technology and make suitable recommendations. The countries view AI technology as a major opportunity for economic growth. They are investing substantially in research \& development in this area. Several countries have entered into collaboration with other countries for R\&D in the area. In the following paragraphs, the steps taken by the government in some of the countries are discussed. The list is not exhaustive. There are many other countries such as Japan, Russia, etc which have been working in the area but are not covered to keep it brief. For instance, Japanese Fifth Generation Computers program was an ambitious AI Program which started in the eighties but could not meet the expectations. However, research in AI has continued in Japan as elsewhere in the world.

\section{United States}

United States has been at the forefront in AI research as well as industry. Government has been funding R\&D in this area since the very beginning. The budget of the US Government for R\&D in AI is at the level of one billion dollars. All the funding agencies such as DARPA and NSF, etc have AIspecific R\&D programmes. Most of the leading universities such as Stanford, MIT, CMU, etc have a 
good number of faculty and research students working in this area. Most of the US-based IT companies have realized the potential of the technology and have developed several AI-based products and services.

United States Government has released three policy documents prepared by the technical experts and other stakeholders. The report titled 'Preparing for the Future of Artificial Intelligence' was released in October 2016 (U.S. Government, 2016). The report has made several recommendations for furthering AI applications. It has also made recommendations on regulations, which need to be enacted in view of the AI powered products like driver-less cars, etc. The report was prepared after an extensive consultation with all the stakeholders through the public workshops co-hosted by Office for Science and Technology Policy (OSTP), industry and academic institutes.

The report titled 'The National Artificial Intelligence Research and Development Strategic Plan'released on 13-10-2016 was prepared by the Networking and Information Technology Research and Development (NITRD) (Frey \& Osborne, 2015). The R\&D Strategy outlined advocates for longterm investments in AI research. Ethical, legal and societal implications of AI should be looked into. It should be ensured that the AI Systems developed are safe and secure. Public Datasets should be developed and shared for AI Training and Testing. Standards and benchmarks should be developed to measure and evaluate AI Technologies. National AI R\&D Workforce Needs should be assessed.

The report titled 'Artificial Intelligence, Automation, and the Economy' released in December 2016 looks into the potential impact of the advances in artificial intelligence (US Government, 2016). The report has recommended for investment in AI for its many benefits. It has also recommended steps for education and training Americans for the jobs which may be available in the future. Further, it has recommended that the Government should aid the workers during the transition and empower them.

\section{United Kingdom}

The UK has been one of the leaders in the area of artificial intelligence. It was Allen M. Turing of the UK who wrote the first paper on the possibility of creating intelligence in computers. He proposed the test called Turing Test which is still referred to as test for AI. Incidently, Turing broke the code of Germans being used in WWII which is regarded as one of the factors for the defeat of Germans. The universities like Edinburgh, Oxford, Cambridge, etc have been conducting research with support from the government since the late fifties. As per a report of UK-RAS, 270 million pound is being invested during 2017-18 and 4.7 billion pounds are planned for research by 2021 as part of UK's industrial strategy (UK-RAS Network, 2017). Recently, the government has announced Industrial Strategy Challenge Fund. 93 million pounds has been earmarked for development of systems which can work in extreme environment required by offshore energy. 38 million pounds is meant for AI and control systems for driverless cars (Hill, 2017).

Science and Technology Committee of the House of Commons has prepared a policy document titled 'Robotics and Artificial Intelligence' (House of Commons Science and Technology Committee, 2016). The Committee has examined various aspects including implications of the advances and adoption for the job market; the extent to which economic opportunities are being exploited in the UK; the steps Government needs to take for funding research and innovation; the steps to address the emerging social, legal and ethical issues. It has made the following broad recommendations under three categories:

Education and Skills - In order to mitigate the consequences of loss of jobs, the Government should ensure that the education and training systems are flexible so that they can adapt to the demands on the workforce and can provide life-long learning. The government should address digital skill crisis through the Digital Strategy. 
Governance: Standards and Regulations - In order to ensure that socially beneficial AI systems are developed, the Government should initiate careful scrutiny of ethical, legal and societal dimensions. Attention should be given to the issues like verification and validation; decision-making transparency, minimising bias, accountability, transparency, privacy and safety. A Standing Commission on AI should be established to examine social, ethical and legal implications of the developments in AI.

Research, Funding and Innovation - Government should design and implement a strategy to develop the skills, securing the investment for future growth in robotics and AI. The government should create Robotics and Autonomous Systems Leadership council to work with the research councils to produce National RAS Strategy to set out the government ambitions and financial support.

\section{China}

State Council of China released the "Next Generation Artificial Intelligence Development Plan" on July 20, 2017 (State Council of China, 2017; Kania , 2017). The document articulates a roadmap for China to become world leader in AI. The three components of the strategy are:

1. Tackling key problems in research and development;

2. Pursuing a range of products and applications;

3. Cultivating an AI industry.

Ministry of Science and Technology of the Chinese will execute the plan through its AI Plan Promotion Office. Under the Plan, China aims to achieve core AI industry of \$22 billion and AI-based industry of $\$ 148$ billion by 2020 . By 2025 , it plans to achieve AI core industry of $\$ 59$ billion and AI related industry of $\$ 740$ billion. By 2030, China aims to create core AI industry of $\$ 148$ billion and AI related industry of $\$ 1.48$ trillion.

The growing technological infrastructure, funding for innovation \& research have led to emergence of several start-up companies. Several IT companies have been able to use the technology in their products and services offerings. The examples are Baidu, Alibaba and Tencent, Turing Robot, DFRobot, Horizon Robotics, etc.

Chinese companies are entering into joint ventures with the high-tech companies of the US. Some of these have established R\&D centres in the US where they are able to attract the available talent. An example is Baidu, which has established Baidu Silicon valley AI Laboratory (SVAIL) in California.

\section{South Korea}

Ministry of Science, ICT and Future Planning (MSIP) of the Government of South Korea is implementing a programme with a budget of 768 million euro and duration of 5 years. The funds are meant for R\&D projects, data infrastructure, and support to industry. Private sector companies such as Samsung, LG Electronics, Hyundai Motors, etc have planned to invest about 1.9 billion euro in AI technology related area (Kania, 2017).

Recently, MSIP has released a document "Mid to Long-Term Master Plan in Preparation for the Intelligent Information society: Managing the Fourth Industrial Revolution" which describes the 
policy of the South Korea Government (Government of South Korea, 2016). The main components of the policy are:
a. Establishment of world-class infrastructure for Intelligent IT
b. Promotion of the application of Intelligent IT to all industries
c. Proactive steps toward reforming and strengthening the social support system

Other initiatives of the Government includes ExoBrian project which was initiated in 2014 with a budget of $\$ 90$ million over a period of nine years. The focus of the project is on natural language processing. The application domains include healthcare, legal and finance. DeepView project, which started in 2015 for nine years focuses on the processing of visual data. One of the activities taken under this project is on building knowledge bases from videos. AI is one of the five thrust areas under Software Star Lab Program. It has been planned to initiate five projects in AI under this program which will provide funds over 8 years (Zhang, 2016).

South Korea is collaborating with other countries including Russia and UK [Korea-UK ICT Cooperation, 2016; Brian-Computer Connection, 2017). Electronics and Telecommunication Research Institute of South Korea and Institute for Information and communication Technology Promotion of Russia are jointly working on brain-computer interface. South Korea is also collaborating with the UK for research in several areas of ICT including AI. Under this, University of Surrey is collaborating with the Institute for Information and Communication Technology Promotion.

\section{Singapore}

Singapore Government has been investing in R\&D in AI for quite some time. It has committed $\$ 450$ million to the National Robotics Programme over a period of three years. Apart from the use of robots in manufacturing, social robots are being developed. An example is Nadine, which is a receptionist robot at the Nanyang Technological University. In the healthcare sector, Singapore Government has established Centre for Healthcare and Robotics Technology (CHART) at the Changi General Hospital.

Singapore Universities have entered into collaboration with the leading educational institutions such as MIT, CMU, etc. It has also started schemes to bring back the Singaporeans living abroad. It is also working on the improvement in the quality of education to prepare its people for new types of jobs.

An important initiative of the Singapore Government in promotion of the startups is the launching of SGInnovate, which has been established to provide support to the entrepreneurs in high technology areas including robotics, AI, etc. It brings the people from the private sector, academia R\&D Centres and Government agencies to work together on innovative solutions.

Singapore is building several AI-powered applications under the initiative 'Smart Nation', which was launched in 2014 (Benner, 2017). The applications are in the sectors ranging from transport to healthcare to public service delivery. In transport sector, Land Transport Authority (LTA) and Nanyang Technological University (NTU) have jointly set up a Transport Research Centre to work on autonomous vehicles including self-driving bus. It is also working on evolving testing standards and certification for the autonomous vehicles. It has already implemented driver-less metro network operational for quite some time. 


\section{INDIAN SCENARIO}

Several startup companies have emerged in India in the recent past (Jain, 2017). According to a report by Zinnov, there are around 170 AI related startups in India and these have received an investment of \$36 million in total (India-based..., 2018). 64 out of these are located in Bengaluru. These are working in the domains of healthcare, e-commerce, finance, etc. Tuplejump, a startup helps the clients visualize the data while taking a decision. It has been acquired by Apple. ClearTax is developing a solution for e-filling using documents directly. AIndra is developing devices with computer vision ability for the applications like facial recognition, detection of cervical cancer, etc. A list of example startups in India is given in Table No. 1.

Edge Networks has developed a solution to match the job profiles with the job seekers. It helps the companies reduce the time and money spent on hiring. Fluid AI has developed a solution to work as gesture controlled assistant. When a customer approaches any product, the system helps with the information just like a human does. It is meant for use in shops to reduce the operational cost. Flutura has developed a solution called Cerebra which collects data on the conditions of the machines and analyzes to advise on the repair schedules, etc. This is expected to reduce the down time of the machine and increase its life. Heckyl collects information on several issues to assist people in stock trading. It is meant for investors, fund managers, brokerage firms, etc. Mad Street Den supports use in searching for products using captured photos.

ShopR360 has developed a video analytics solution which can be used to analyze CCTV videos. It can distinguish staff from the customers and helps in the strategic placements of the products in the stores. Niki.ai has developed a solution which can be used for developing conversational assistants for placing orders like booking cabs, payment of utility bills. The client list of Niki.ai includes HDFC Bank, Oxigen Wallet, Ticketgoose. SigTuple is focussing on the affordable solutions for medical diagnosis using AI a microscope and a cell phone. Socialcops develops solutions to assist in decision-making. It assists by visualization of the collected data. PHRAZOR from Vphrase converts structured data such as graphs into natural language sentences. This is used for automated generation of reports on various topics.

Table 1. Indian Startups in Artificial Intelligence

\begin{tabular}{ccc}
\hline Startup & URL & Task \\
\hline Edge Networks & www.edgenetworks.in & Matches the job seekers with jobs available \\
\hline Fluid AI & www.fluid.ai & Provides customer information on the products in an interactive way \\
\hline Flutura & www.flutura.com & Monitors health of machines to advise on maintenance \\
\hline Heckyl & www.heckyl.com & Analyzes stock related information to advises on stock trading \\
\hline Mad Street Den & www.vue.ai & Helps customers locate products using captured photos \\
\hline ShopR360 & www.shopr360.com & Video analytics solution which can distinguish staff and customers \\
\hline SigTuple & www.sigtuple.com & Affordable diagnosis solution using a microscope, cell phone and cloud \\
\hline Social Cops & www.socialcops.com & Interpretation of data \\
\hline VPhrase & www.vphrase.com & Converts structured data such as graphs, etc into words \\
\hline Freenome & www.zephyrhealth.com & Analyzes genetic material to find disease signature at an early stage \\
\hline
\end{tabular}


IT majors like TCS, Infosys, etc are also developing AI-based solutions for their needs. TCS has developed a Virtual Assistant which can interact with the customers regarding insurance products (Tata Consultancy Services, 2012). It can interact in spoken natural language. Infosys has automated several IT support processes using AI-based solutions. IBM has deployed Watson for Oncology product in some of the hospitals for use in the treatment of cancer patients (Manipal Hospitals..., 2018). Recently, Cyril Amarchand Mangaldas, a reputed law firm has announced that it has entered into collaboration with Kira, a Canadian firm offering AI-based solutions for law firms.

Government has been spending on R\&D in AI for quite some time. MeitY (erstwhile DOE) started knowledge-based computer Systems Project in 1986 with financial support from UNDP. Several development activities were done under the project. The project created necessary infrastructure in several academic institutions / R\&D centres like C-DAC, etc. Later on, a programme called National Programme on Perception Engineering was initiated by the Ministry. Some prototype systems such as robotic arms, etc have been developed under the programme. Some projects were also taken up under Technology Development for Indian languages Programme of the Ministry. A large percentage of funds have been spent on machine translation, text-to-speech, and speech-to-text systems.

DRDO has been funding AI projects at Centre for Artificial Intelligence and Robotics (CAIR) for defence as well as civil applications. Funding of R\&D by industry has been limited to few companies only. However, the situation is changing now. Recently, Infosys has provided Rs. 50 million to Inderprastha Institute of Information Technology (IIIT) Delhi for research in AI.

The impact on the job opportunity would be more serious in India due to the high percentage of the people employed in low-skill jobs. A large number of people are employed in the BPO type of services which are most likely to be affected. Similarly, the demand of IT professionals, especially those doing routine white collar jobs, is bound to fall due to automation of various tasks. As these areas have been providing jobs to millions of the graduates during the last two decades, the situation is alarming and need immediate attention from the stakeholders.

Recently, some companies have announced that they are releasing the workers or hiring less number of workers. As mentioned earlier, Infosys has announced that it had released 9000 IT professionals ${ }^{6}$. Similar announcements have been made by some other companies. The official reason for the layoffs being given by these companies is poor performance of the employees but this has been denied at various forums. Though loss of jobs may happen due to automation, a good number of jobs are likely to be added as the economy in India is growing at a high rate.

\section{WAY FORWARD}

India has a unique opportunity at this moment. Using the talent available within the country, it can repeat the success story of IT industry. At the same time, if necessary steps are not taken in time, it will lose the opportunity. AI can help in the major programmes of the Government viz. Digital India, Make in India, and Skill India (Vempati, 2016; Ghosh \& Mitra, 2017). In order to accelerate development of AI technology and its applications, it is necessary to take steps for Applications \& Infrastructure Development, Policy \& Regulations, Research \& Development and Human Resource Development as illustrated in the Figure No. 1. These are discussed in the following sections.

$6 \mathrm{http} / / / w w w . b u s i n e s s t o d a y . i n / c u r r e n t / c o r p o r a t e / i n f o s y s-r e l e a s e s-9000-e m p l o y e e s-i n-t h e-p a s t-o n e-y e a r / s t o r y / 244596$. html [Accessed February 20, 2018] 


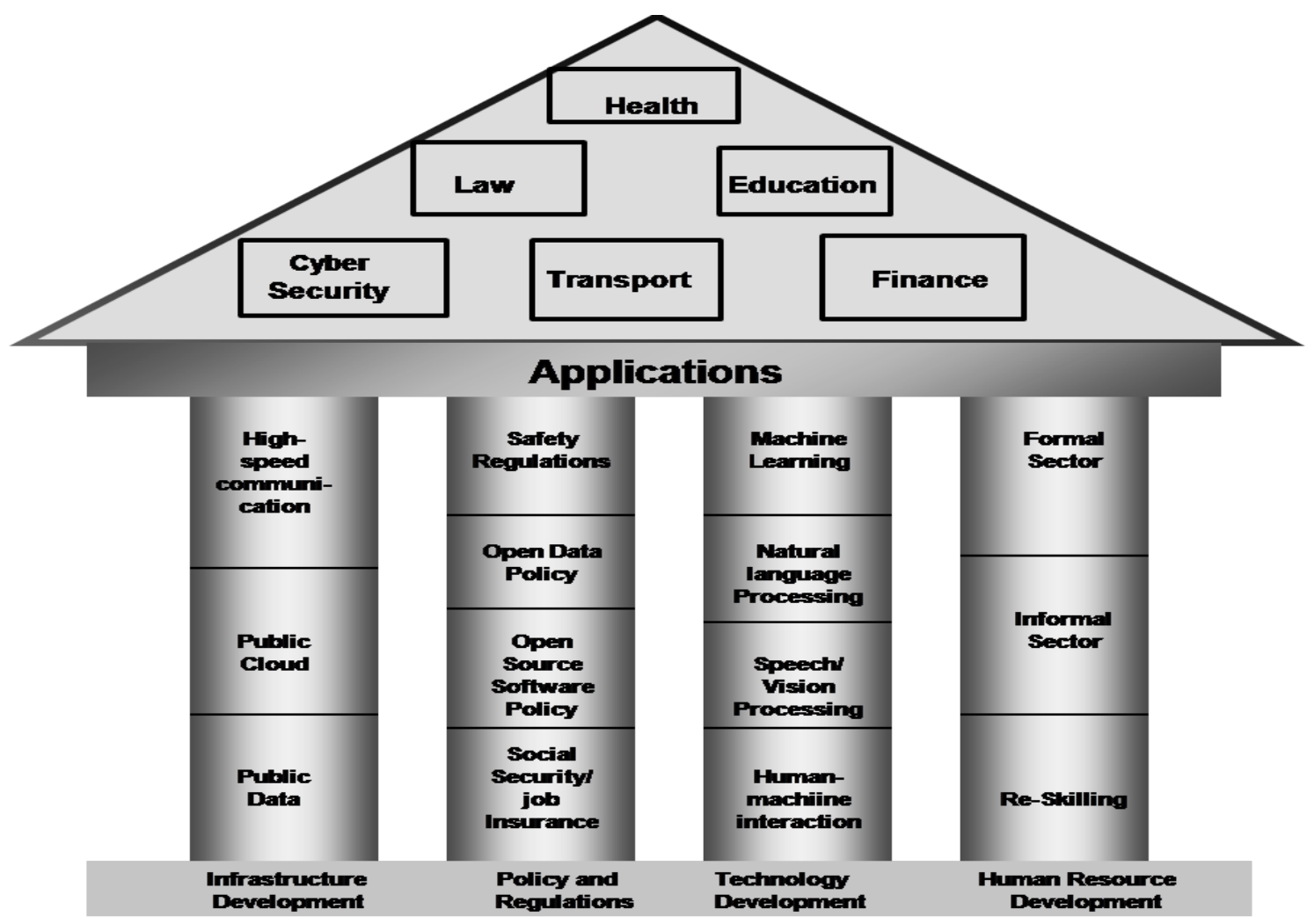

Figure 1. Way forward for India

\section{Applications and Infrastructure Development}

As in other countries, India can gain significantly by the adoption of AI technology. Most of the applications developed elsewhere in the world can be developed in India as well. However, the applications have to be customized for the local needs. Few example applications are discussed below.

A virtual nurse can be developed to share the workloads of the human nurses. Due to lack of human resource in the public healthcare facilities in India, a nurse is often overloaded. As she has to attend several persons, there is always possibility of the lapses. For example, after the treatment has been completed and the patient is being discharged, a nurse is supposed to guide the patient and the caretakers on the precautions to be taken after leaving the hospital and between the follow up consultations. A large number of people suffer from the complications after the treatment as they are not informed properly on the precautions which have to be taken. This type of information can be provided to the patients using an AI-based system.

Similarly, a chatbot can be developed to advise the patients on several health-related matters in natural language. The patients don't like to discuss about several types of diseases like HIV, STDs, tuberculosis, etc as the patients suffering from such diseases are treated as untouchable in the society. Such applications can help in this case. However, they would be happy to consult a virtual medical counsellor. In India, mental illness is often not treated due to lack of awareness. Fortunately, the country has a high level of mobile penetration. Such applications can be easily made available on mobile devices.

Applications like DoNotPay can be used to help people by providing relevant legal information. Such applications are quite relevant for Indian society where a large percentage of the population is ignorant about the laws and procedures concerned with day-to-day activities. 
Some of the areas where such applications can be developed include dowry matters, domestic violence, consumer rights protection, violence against children, taxation, etc. To start with, systems with natural language interface in English can be developed. Later on, it can be extended to other languages, and finally to speech-based systems.

AI-based education systems or intelligent tutoring systems will be useful in improving the quality of education by the existing teachers, especially in professional education. Shortage of meritorious teachers is a common problem in all types and levels of education in the country due to several reasons. Use of natural language processing makes it possible for the student to interact with the application in natural language. AI-based system is not expected to replace human teacher but can be used to provide supplementary information.

The government must create infrastructure to support development of AI applications. One critical infrastructure is cloud which is needed for the development of applications. AI applications require high computational power, large memory and storage space which are available on the cloud. Some systems which were possible but could not be built due to the unavailability of cloud infrastructure earlier have been implemented on the cloud. Google translation system has become possible only due to the availability of cloud. Several AI applications use public data which is not supposed to be stored or processed outside the country. If one uses cloud space from any vendor like Amazon, Microsoft, etc, the data may be hosted abroad, which is not desirable due to several reasons.

AI applications for public goods can be developed only if we have adequate infrastructure for making it available to the developers. Often public data is not made available for privacy reasons. However, such data can be annonymized before making it available. High speed network is another requirement necessary for development of AI applications. This is essential to collect and share large amount of data. Though connectivity has become available in urban areas, it remains a problem for rural and remote areas.

\section{Regulations and Policy}

As AI applications touch several aspects of human life, regulations are needed to ensure safety of the people, protection of privacy, etc. For instance, in the area of transport, if autonomous vehicles are to be permitted on the roads or air, regulations are needed to ensure public safety. A self-driving car must take care of enormous number of possible situations on the road. While deciding the permission to use the autonomous vehicles, it is necessary to assess the potential risks in both the situations i.e. when conventional vehicles are used and when AVs are used.

Regulations may be linked to the performance of the products. In this case, further use depends on the performance. If AI-based applications / services are found to be safer than human-based applications/services, more use may be permitted. If it is found to be less safe, the use should be restricted till the further development of technology.

Regulations are needed to permit the use of AI in the critical domains like healthcare where the autonomous systems are expected to advice on the diagnosis and treatment which may affect the recovery of the patient. At the moment, people are often not comfortable with the machines taking such decisions with major implications. In the beginning, it may be necessary to keep human doctor in the loop so that the decisions could be reviewed.

Regulations need to be made to ensure that the applications developed are not biased towards a specific view. The biasing may be intentional when it is incorporated by the developer of the application. Sometimes, it may be incorporated due to the training data set. The developer may not do it intentionally. 
Policy is needed to make the public data available to the developers to promote the development of applications. Several applications depend on the availability of large amount of public data. For example, the data on the traffic, road conditions may be necessary to develop applications for advising the drivers on the routes. It may be necessary to annonymize the data before making it public in order to protect the privacy of the individuals and organizations. This is especially important in the areas like healthcare where the leakage of personally identifiable data may hurt the social reputation of the person. Government in several countries have already made the public data available to the developers under certain terms and conditions.

Policy is needed for making the results of R\&D available to the public. Several R\&D projects are funded by the Government in the country but often the results remain confined to a limited number of persons. Several countries have made legislations to make the results of the R\&D funded by the Government available to the public by putting it in open-source domain. This ensures that the benefit of the public money reaches the public. A similar policy is needed in the country.

As a policy, Government should also work on making people aware about this technology. This is necessary if we want to create confidence in the people for using AI-based applications. It is more important in the applications like medicine where people may not feel comfortable in following the advice of a machine.

Policy is needed to handle the impact on the job opportunity. Several studies indicate that the use of AI is likely to reduce the job opportunity in several sectors. Infrastructure and policy are needed to retrain the workers to enable them take up new jobs which may emerge. However, it may not be always possible to retrain the manpower to take up the new emerging jobs immediately. Therefore, policy is needed to provide some type of social security to the workers who may be displaced.

As India does not have a comprehensive social security system like developed countries, this may lead to chaos if necessary steps are not taken in time. The employers should be persuaded to have a policy for retraining the workers who are likely to be displaced. If that is not possible, the employer should provide a good severance compensation which is sufficient for the worker during the transition period. Alternately, the companies may be required to make adequate contribution to any insurance plan which can provide support to the worker during the transition period.

\section{Research and Development}

Government has a major role to play in funding R\&D and innovation projects. Most of the developed and several developing countries are investing heavily in R\&D and innovation in the area of artificial intelligence. India needs to initiate a Programme to support R\&D and innovation in this area.

Involvement of industry is necessary while funding R\&D projects. A major problem faced in India is that the $R \& D$ results don't lead to commercial products. The main reason of this situation is the absence of interaction between the R\&D centres/academic institutions and industry. In order to get the input from the industry, it is necessary to hold discussions with them through regular workshops/ meetings. Industry associations can play a role in this matter. They can provide a consolidated view of the companies on the issues.

The companies need to be persuaded to form a consortium and so that a common fund could be made available to support the projects at the R\&D centres / academic institutions for carrying out research and development on the topics of common interest. Government agencies may provide complementary financial support to these R\&D centres / academic institutions in the high risk projects. 
Feasibility studies need to be conducted before initiating R\&D or application development projects. The success of AI projects depends on the tasks and domains. Before funding R\&D or application development projects, it is necessary to conduct some feasibility studies. Once the feasibility study has been completed, pilot level or demonstration projects should be initiated before large scale deployment projects.

Centres of Excellence should be created in the academic institutions and R\&D centres. Industry should be invited to collaborate with these centres to work on the technology and applications of their interest. Apart from R\&D of long-term benefits, these centres should work on the ideas which can lead to startup companies.

Several AI applications have been developed for national security. So far, most of the funding in R\&D in this area has come from defence. In the US, DARPA has been the main sponsor of R\&D in this area. In India, Centre for Artificial Intelligence and Robotics (CAIR) has been working on the projects relevant to national security. This needs to be strengthened further.

Despite all the achievements in IT, a major part of the population in the country has not been able to get the benefits due to the language problem. Most of the applications are in English which keeps them at disadvantage. There is a need of developing technology to provide equivalent information search engine for Indian languages. An example can be seen in China. It has been done for Chinese language by Baidu, a start-up company.

International cooperation in $\mathrm{R} \& \mathrm{D}$ in this area is important for India as it can provide an advantage to the country which is lagging behind the developed in high technology. International collaboration in this area is quite common among the developed countries as well. It is not difficult to find the countries willing for cooperation in this area. Apart from the technologically advanced countries like USA, UK, etc, India should also explore possibility of collaboration with smaller economies like Brazil, Portugal, etc. These countries often face the problems similar to India and therefore, the collaboration would be quite useful. Academic institutions such IITs, IIITs, etc should be involved in the R\&D collaboration.

\section{Human Resource Development}

In order to cope up with the problems due to the loss of jobs, the workforce will have to be retrained to take up new types of jobs which may emerge with the automation of the processes. As the people lose jobs, they must be retrained and re-employed by providing the necessary training. For this, there is a need to look into education and training infrastructure and re-align it with the needs of the present day. Both formal and informal education systems should be reviewed to produce the manpower who can deal with the changing needs of the society.

Indian startup companies find it quite difficult to recruit engineers with AI background (Punit, 2018). Several steps need to be taken. There is a need to initiate educational programmes in this area. Most of the universities in the developed countries offer graduate programmes in AI. In contrast to that, very few universities in India offer programmes in AI. Some educational institutions offer AI as one subject in the B. Tech. or M. Tech. Programme. This is not sufficient in the field like AI. The universities and technical education institutions need to be supported by the Government for initiation of these programmes.

There is a need to attract bright students to do research in the area of AI. Doctoral and Postdoctoral fellowships should be instituted and made available to the people interested in research in the area of AI. The fellowships should carry higher stipend and other benefits to attract the talented students. These students should be encouraged to work in the areas which can lead to useful applications. 


\section{CONCLUSIONS}

India has a unique opportunity to apply the technology to solve some of its biggest problems such as shortage of healthcare facility, low quality of education, etc. It is not possible to meet the target of providing good healthcare or quality education using conventional methods. For instance, the number of doctors needed to to provide good quality healthcare is so large that it cannot be achieved in several years. AI technology provides an alternative to achieve the same.

The report has reviewed international as well as national status of adoption of artificial intelligence technology. While the technology has potential to increase the economic growth rate considerably, it is likely to impact the job opportunity adversely. The challenge before any country is to make the best use of opportunity while dealing with the job loss issue, simultaneously. The report has examined the developments in some other countries to find the steps taken by them.

Based on these, it has suggested a way forward for India, which involves infrastructure development, policy \& regulations, research \& development, and human resource development. All the stakeholders need to come together to discuss on these issues. Government has a major role to play in infrastructure development, applications in public sector, policy \& regulations, technology development and HRD. However, these can be successfully done with the support from industry.

Though the report has brought out the major issues, it is necessary to institute some studies to collect the precise information for deciding the steps on infrastructure development, policy \& regulations, and technology development, etc. It is also necessary to conduct a survey on the expected loss of jobs in various sectors so that appropriate policy could be framed. Such studies can provide the basis for policy responses from the government. Though it may be early to formulate new regulations, the existing regulations need to be reviewed and modified, if necessary.

\section{DISCLAIMER}

The views expressed in this report are those of the author in his individual capacity and do not reflect the official views of Government of India, Ministry of Electronics and Information Technology (MeitY).

\section{REFERENCES}

Domingos, Pedro. The Master Algorithm: How the Quest for Ultimate Learning Machine will Remake Our World. Basic Books. 2015

Purdy, Mark and Daugherty Paul. Why Artificial Intelligence is the Future of Growth. Accenture. 2016

U.S. Government, Executive Office of the President, NSTC Committee on Technology. Preparing for the Future of artificial Intelligence. October 2016

U.S. Government, Executive Office of the President. Artificial Intelligence, Automation and the Economy. December 2016

House of Commons Science and Technology Committee. Robotics and Artificial Intelligence. September 2016. 
State Council of China. New Generation artificial Intelligence Development Plan. State Council Document No. 35. July 2017

Government of South Korea, Ministry of Science, ICT and Future Planning. Mid-to Long-Term Master Plan Preparation for the Intelligent Information Society: Managing the Fourth Industrial Revolution. Policy Document. 2016

Benner, Tom. Singapore: A Smart Living Laboratory. Scientific American. 2017.

Nassiri-Mofakham, Faria (Ed.). Current and Future Developments in Artificial Intelligence, Vol. 1. Bentham Science Publishers. 2017

Houlihan, David. ROSS Intelligence \& Artificial Intelligence in Legal Research. Blue Hill Research. 2017

Mills, Michael. Artificial Intelligence in Law: The State of Play 2016. Thomson Reuters Legal executive Institute. 2016

How artificial intelligence is transforming the legal profession. http://www.abajournal.com/magazine/article/ how_artificial_intelligence_is_transforming_the_legal_professional [Accessed February 20, 2018]

Cessna, Abby. Future of AI and Law. Cornell Law School. 2015

RAVN Systems. The Power of Understanding Artificial Intelligence in the Legal World. White Paper. 2016

DoNotPay. http://www.donotpay.co.uk/ [Accessed February 20, 2018]

e-Brevia. Contract Analyzer. https://ebrevia.com/ebrevia-contract-manager [Accessed February 20, 2018]

Kira Systems. Kira for Contract Analysis. https://www.kirasystems.com/how-it-works/contract-analysis [Accessed February 20, 2018]

Yacoub, Husein Nuseibeh. Artificial Intelligence: Opportunities and challenges in finance industry. Gulf News. http://gulfnews.com/business/sectors/banking/artificial-intelligence-opportunities-and-challengesin-finance-industry-1.1976801 [Accessed February 20, 2018]

Satariano, Adam. Silicon Valley Hedge Fund Takes On Wall Street With AI Trader. https://www.bloomberg. com/news/articles/2017-02-06/silicon-valley-hedge-fund-takes-on-wall-street-with-ai-trader [Accessed February 20, 2018]

Desai, Falguni. The Age of Artificial Intelligence in Fintech. https://www.forbes.com/sites/ falgunidesai/2016/06/30/the-age-of-artificial-intelligence-in-fintech/\#269da1a15028 [Accessed February 20, 2018]

Busayo Ogunsanya. AskMyUncleSam. http://venturebeat.com/2017/01/27/how-this-chatbot-powered-bymachine-learning-can-help-with-your-taxes/ [Accessed February 20, 2018]

Brin, Sergy and Page, Lawrence. The Anatomy of a large Scale Hypertextual Web search Engine. Proc. 7th Int'l World Wide Web Conf. (WWW98), ACM Press, New York, 1998

Google Inc. Marketing and Advertising Using Google. http://static.googleusercontent.com/media/www. google.com/en//intl/ar/googlebooks/pdf/MarketingAndAdvertisingUsingGoogle.pdf [Accessed February 20, 2018]

Jiang, Tao et. el. Self-Driving Cars: Disruptive or Incremental? Applied Innovation Review. June, 2015

Alluhaidan, Ala, "Recommender System Using Collaborative Filtering Algorithm" (2013). Technical Library. http://scholarworks.gvsu.edu/cistechlib/155 [Accessed February 20, 2018]

Perez, Sarah. Starbucks unveils a virtual assistant that takes your order via messaging or voice. https:// techcrunch.com/2017/01/30/starbucks-unveils-a-virtual-assistant-that-takes-your-order-via-messaging-orvoice/ [Accessed February 20, 2018]

Amelia. http://www.ipsoft.com/amelia/ [Accessed February 20, 2018] 
Amazon Go Store lets shoppers pick up and walk out. The Guardian. https://www.theguardian.com/ business/2016/dec/05/amazon-go-store-seattle-checkouts-account [Accessed February 20, 2018]

Jucikas, Tadas. Artificial Intelligence and the Future of Energy. https://medium.com/wepower/artificialintelligence-and-the-future-of-energy-105ac6053de4 [Accessed February 20, 2018]

Agrawal, Ajay; Gans, Joshua and Goldfarb, Avi. How AI will Change Strategy: A Thought Experiment. Harvard Business Review. https:/hbr.org/2017/10/how-ai-will-change-strategy-a-thought-experiment [Accessed February 20, 2018]

Government of Canada. Scan of Emerging Issues: The Changing Nature of Work. Policy Horizons Canada.

McKinsey Global Institute. Disruptive technologies: Advances that will transform life, business, and the global economy. 2013

Frey, Carl and Osborne, Michael. Technology at Work: The Future of Innovation and Employment. Citi Report. February, 2015.

U.S. Government, Executive Office of the President, NSTC Committee on Networking and Information Technology Research and Development. The National Artificial Intelligence Research and Development Strategic Plan. October 2016

UK-RAS Network. Artificial Intelligence and Robotics. White Paper, 2017.

Hill, Rebecca. Gov.UK pops open tin of AI and robotics research cash. The Register. June 22, 2017

Kania, Elsa. China’s Artificial Intelligence Revolution. The Diplomat. July 22, 2017

Ha, Jeong Eun. Artificial intelligence industry in South Korea. Rijksdienst voor Ondernemend, Nederland, 2016.

Zhang, Byoung-Tak. Human and Machines in the Evolution of AI in Korea. AI Magazine, 2016.

Korea-UK ICT Cooperation: S. Korea, UK to Work Together More Closely in ICT Industry including 5G. Business Korea. September 13, 2016

Brian-Computer Connection: S. Korea, Russia to Conduct Joint Research on Brain-Computer Connection Technology. Business Korea. August 17, 2017

Jain, Sumit et al. India's Tryst with Artificial Intelligence. Kalaarri Capital. 2017

India-based AI startups draw attention of tech biggies like Apple, Facebook. http://economictimes.indiatimes. com/small-biz/startups/india-based-ai-startups-draw-attention-of-tech-biggies-like-apple-facebook/ articleshow/55589444.cms [Accessed February 20, 2018]

Tata Consultancy Services. Application of Artificial Intelligence in Insurance Customer Interactions. White Paper. 2012

Manipal Hospitals announces national launch of IBM Watson for Oncology. https://watsononcology. manipalhospitals.com/uploads/press_releases/Watson_4_-_APN_News.pdf [Accessed February 20, 2018]

Vempati, Shashi Shekhar. India and the Artificial Intelligence Revolution. Carnegie India. August 2016

Ghosh, Sudipta and Mitra, Indranil. Artificial Intelligence and Robotics - 2017: Leveraging artificial intelligence and robotics for sustainable growth. ASSOCHAM-PWC Report. March, 2017

Punit, Itika Sharma. Why are Indian engineers so afraid of 'artificial intelligence'? https://scroll.in/ article/829652/why-are-indian-engineers-so-afraid-of-artificial-intelligence [Accessed February 20, 2018] 